\title{
LISTENERS' ATTITUDES: SPEECH SUPPLEMENTA- TION STRATEGIES FOR IMPROVING EFFECTIVE- NESS OF SPEAKERS WITH MIXED DYSARTHRIA AS A RESULT OF MOTOR NEURON DISEASE
}

\author{
Natalie Toy and Karin Joubert \\ Department of Speech Pathology and Audiology, University of the Witwatersrand, South Africa
}

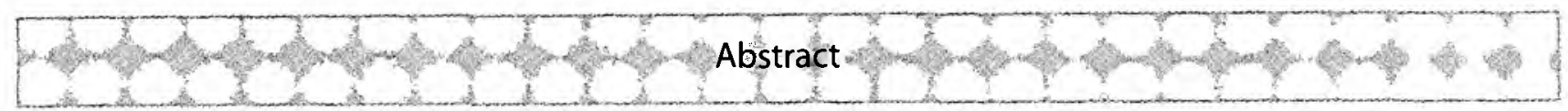

This study examined unfamiliar and familiar listener attitudes towards the use of combined alphabet-topic cues and a control condition (habitual speech with no cues) associated with the speech of three individuals with severe mixed dysarthria. Twolistenergroups $(\mathrm{N}=36)$ were shown experimentally imposed visual images of the combined al phabet-topiccuestrategyin conjunction with recorded auditory presentations with the habitual speech of three individuals with mixed dysarthria. Using a 7-point Likert scale, listeners were asked toratehow effective they thought the speakers communicated; how comfortable theywere communicating with the speakers; and how persistent they were in trying to understand the speakers. The results revealed that therewerenosignificant differences in the attituderatings of familiarlisteners as compared tounfamiliar listeners. However, resultsrevealed thatrating of communicativeeffectiveness, comfortcommunicating with speakers and listener persistence were each more favourable when using the combined cue condition than purelyhabitualspeech. Theresultssuggestthataugmentativeandalternativecommunicationstrategiesproviding frequent and specific cues regarding the content and constituent words of a message may enhance the attitudes of listeners.

Keywords: Dysarthria, intelligibility, listener attitudes, speech supplementation, augmentative and alternative communication.

$\mathrm{S}$ peech, one of the pre-dominant forms of communication is used in every facet of our lives, whether for the purpose of expressing our basic needs, social interaction or vocational purposes (Light, McNaughton, Gulens, Kresman, Williams \& Cohen, 1999). The inability to communicate via speech has significant psychosocial consequences on an individual's functioning and role in society, such as alterations in social and family roles, as well as family interactions (Fox, Sohlberg \& Fried-Oken, 1999).

In addition, Britnell, Madill, Montgomerie and Stewin (1992) found that individuals with a disability reported decreased participation in occupational roles and recreational activities. Individuals with Motor Neuron Disease (MND) reported that as a result of a reduction in their motor abilities and speech intelligibility, attitudes changed from kindness to awkwardness and avoidance (Mathy, Yorkston \& Gutmann, 2000), contributing to decreased participation in various social activities and eventually isolation.

Dysarthria, a deficit of speech production, re- sults from damage to the peripheral or central nervous system and is characterized by slowness in coordination and imprecision of the movement of the speech musculature. Reduced speech intelligibility is a hallmark characteristic of most dysarthrias and has a significant impact on an individual's ability to communicate, thus ultimately affecting an individual's quality of life (Hustad \& Beukelman, 2001). It has been estimated that dysarthria accounts for up to $54 \%$ of all communication disorders associated with stroke, traumatic brain injury and progressive neurological disorders such as MND, Parkinson's disease and Multiple Sclerosis (Duffy, 2005).

MND, also known as Lou Gehrig's disease or Amyotrophic Lateral Sclerosis, is a progressive neurological disease, which usually results in severe physical disability involving all four limbs, speech, swal-

Author Contact: Department of Speech Pathology and Audiology

Private Bag 3,

Wits, 2050, South Africa

E-mail: Karin.Joubert@wits.ac.za 
lowing and breathing (Young \& McNicoll, 1998). The cause of MND is unknown and is said to occur in 1.5 per 100,000 of the population worldwide with the average age of onset at 65 years and a greater incidence in males (Freed, 2000). Patients with MND usually do not show any changes in cognitive abilities (Young \& McNicoll, 1998). The disorder is characterized by deficits in either the lower motor neuron or the upper motor neuron, but usually results from a combination of both (Freed, 2000).

The type of dysarthria that occurs in individuals with MND depends on which motor neurons are affected. In the initial, mild stages, individuals with lower motor neuron involvement will present with flaccid dysarthria, whereas those with upper motor involvement present with spastic dysarthria. However, as the disease progresses to involve both the upper and lower motor neurons, patients will present with mixed dysarthria that will predominate throughout most of the disorder (Freed, 2000).

In a progressive neurological disorder such as MND, approximately $75 \%$ of the patients will reach a point where intelligible verbal communication is no longer possible (Freed, 2000). The rapid decline of speech function in patients with MND is common, but is not inevitable. Mathy et al. (2000) identified and documented the progression of dysarthria in individuals with MND (Appendix A). In the early stage (Stage 1), there are no obvious deficits noticed in an individual's speech, but as the disease progresses, the individual's speech intelligibility becomes more compromised until at the final $5^{\text {th }}$ stage, individuals with MND lose all speech function.

Communication by nature requires the involvement of two communication partners who take turns playing the roles of the listener and speaker throughout the course of interaction (Hustad \& Beukelman, 2002). Speech intelligibility can be defined as the extent to which a spoken utterance is understood by the listener (Yunusova, Weismer, Kent $\&$ Rusche, 2005) and is seen as a significant factor in determining whether an individual is an effective communicator within a conversation (DePaul \& Kent, 2000). Intelligibility is therefore a central concept in the field of speech-language pathology, particularly for speakers with dysarthria, whose compromised intelligibility may impair their ability to communicate effectively in their daily lives (Hustad, Jones \& Dailey, 2003).

Sentence intelligibility measures are often used for clinical purposes such as quantifying the severity of dysarthria, measuring progress throughout the intervention process and in determining a speaker's communicative effectiveness (Hustad \& Cahill, 2003). In addition, intelligibility measures are also used for basing decisions on the implementation of alternative and augmentative communication (AAC) strategies (DePaul \& Kent, 2000).

Speech intelligibility is an interactive process that fluctuates for any given speaker and is dependent on a host of variables pertaining not only to the speaker, but also to variables associated with the listener and the communicative context (Hustad \& Cahill, 2003). Relatively few of these variables that are thought to have an influence on intelligibility, have been systematically studied. However, recent research is beginning to demonstrate the importance of "signal-independent information" extrinsic variables on intelligibility (Hustad \& Cahill, 2003). These extrinsic variables are not dependent on the acoustic signal produced by the speaker, but focus on factors that influence intelligibility for the listener.

There is a growing body of experimental research suggesting that AAC strategies designed to supplement dysarthric speech can significantly increase speech intelligibility for individuals with dysarthria (Beukelman \& Yorkston, 1977; Crow \& Enderby, 1989; Hunter, Pring \& Martin, 1991; Hustad \& Beukelman, 2001). The use of low technology AAC strategies in progressive diseases such as MND has received significant attention in recent years (Beliveau, Hodge \& Hagler, 1995; Dongilli, 1994; Garcia \& Cannito, 1996; Hustad \& Beukelman, 2001). These strategies include alphabet supplementation, topic supplementation and combined supplementation.

The alphabet supplementation strategy involves the use of an alphabet board that speakers point to, to indicate the first letter of each word while simultaneously speaking it (Beukelman \& Yorkston, 1977; Crow \& Enderby, 1989; Hustad \& Beukelman, 2001; Yorkston, Beukelman, Strand \& Bell, 1999). This strategy aims to improve intelligibility by providing the listener with knowledge regarding the phonetic content of a word. In alphabet cues, the listener receives first-letter-of-word orthographic information that serves to limit the number of possible word choices and therefore improve the chances of correct word identification (Hustad, 2001a; Hustad \& Beukelman, 2001; Hustad \& Garcia, 2005). Several studies have demonstrated that alphabet supplementation can lead to sentence intelligibility scores that average approximately $35 \%$ higher than intelligibility scores associated with habitual speech (Beukelman \& Yorkston, 1977; Beukelman et al., 2000; Crow \& Enderby, 1989; Hustad et al., 2003).

With topic supplementation strategies, the use of a communication board containing key words or phrases that represent topics or contexts in the speaker's life is used (Hustad, 2001a). Topics are represented orthographically or through the use of picture symbols. The speaker would point to the topic of his/her message before producing it verbally (Beukelman et al., 2002; Hustad, 2001b). Hustad and Beukelman $(2001,2002)$ suggest that this strategy assists the listener to narrow the expectations of the semantic content of the message. The topic supplementation strategy has been shown; to produce sentence intelligibility scores ranging from $3 \%$, to $16 \%$ higher than habitual speech (Beukelman \& Yorkston, 1977; Beukelman et al., 2002; Crow \& Enderby, 1989; Hustad et al., 2003).

The combined supplementation strategy involves the integration of both alphabet and topic supplementation strategies. In this scenario, the speaker first presents the listener with a topic cue followed by a first-letter cue for each word spoken. This strategy has been shown to provide the highest sentence intelligibility scores, yielding as much as $35 \%$ to $40 \%$ higher scores than habitual speech (Beukelman \& Yorkston, 1977; Beukelman et al., 2000; Crow \& Enderby, 1989; Hustad et al., 2003).

The concept of 'attitude' has important social implications for individuals who use speech supplementation strat- 
egies to enhance their intelligibility. Attitudes have been a topic of interest within the disability literature for the past five decades (Hustad \& Gearhart, 2004). The widely accepted notion of the AAC acceptance model states that attitude is a concept made up of three different components, namely affective, cognitive and behavioural components (Antonak \& Livneh, 1988; Eiser, 1986; Greenwald, Brock \& Ostrom, 1968; Lasker \& Bedrosian, 2000; Triandis, Adamopoulos $\&$ Brinberg, 1984). The affective component of attitude involves emotional and physiological reactions to AAC such as comfort level or frustration. The behavioural component relates to the willingness to use $A A C$ or to interact with someone using AAC, whilst the cognitive component of attitude relates to both the speaker and partner's perceptions of communication skills (Hustad, 2001b).

The attitudes of potential communication partners may be influenced by factors such as rate, intelligibility, personality characteristics and their view on disability. These attitudes in turn, may impact on a speaker's desire or motivation to adopt one of the previously mentioned speech supplementation strategies and hence provides valuable information to support clinical decisions (Hustad \& Gearhart, 2004).

Hustad and Gearhart (2004) investigated 168 listeners' attitudes towards 7 dysarthric speakers who implemented three different supplementation strategies (topic/subject cues, alphabet cues and combined topic-alphabet cues) by measuring ratings relating to cognitive, affective and behavioural components of attitude. The results showed that ratings for the behavioural component were the highest for all but one speaker. The study concluded that there is strong evidence of a positive relationship between intelligibility scores and attitude ratings, with attitude ratings increasing linearly with intelligibility scores.

In addition the study yielded important clinical implications for individuals who supplement their speech with AAC strategies. The implementation of combined cues or alphabet cues resulted in higher attitude ratings relative to topic cues and habitual speech. These strategies also resulted in the greatest intelligibility gains (Hustad et al., 2003). As listeners appeared to be more willing to communicate with speakers who implemented alphabet or the combined cues strategies, the use of strategies aimed at enhancing intelligibility will likely serve to particularly enhance the behavioural attitude of listeners.

Also of particular interest in recent studies is the means of defining the optimal listener-speaker pair. Familiarity has been among one of the many factors used in explaining variations in listener performance (DePaul \& Kent, 2000).

Familiarity is however a vague and abstract concept, and for research purposes needs to be usable and measurable based on definable characteristics. In a longitudinal case study, DePaul and Kent (2000) studied the effect of listener familiarity and proficiency on intelligibility judgements of individuals with MND. The results indicated better performances for familiar listeners as opposed to unfamiliar listeners. Familiar listeners were defined as individuals who had experience in communicating, on a daily basis with a person with a communication disorder. Unfamiliar listeners were defined as individuals that had no more than incidental experience listening to and communicating with persons having communication disorders. The results of this study suggest that speech-language pathologists should use listener training as part of their practice standards in dysarthria treatment. The components should include: (i) establishing pre-treatment listener's proficiency, (ii) training listeners through familiarization techniques, and (iii) re-assessing intelligibility using these trained listeners.

Additional research examining familiarity with dysarthric speech is required in order to obtain conclusive results regarding the influence of familiarity on the speech intelligibility of individuals with dysarthria.

This study is largely based on the previous work conducted by Hustad (2001b), which investigated 68 unfamiliar listeners' evaluation of speech supplementation strategies used for severely dysarthric speech. As communication is dyadic (Hustad \& Beukelman, 2002), requiring interaction between the speaker and listener in communicative exchanges, speech intelligibility and listener attitude is a central construct in the area of AAC (Hustad, 2001a,b). It is therefore of significance to determine listeners' attitudes towards $\mathrm{AAC}$ and in particular speech supplementation strategies, as this can greatly influence the acceptance of AAC systems and strategies by the user.

\section{Method}

\section{Aim}

The primary aim of the study was to compare unfamiliar and familiar listeners' attitudes towards the use of speech supplementation strategies (combined topic and alphabet cues) by speakers with mixed dysarthria. Three sub-aims delineate the means by which the primary aim of the study was realised:

- To describe the effects of speech supplementation strategies on listeners' perception of communication effectiveness.

- To describe the effects of speech supplementation strategies on listeners' willingness to interact with speakers.

- To describe the effects of speech supplementation strategies on listeners' ratings of persistence in trying to understand speakers.

\section{Research Hypotheses}

As the research investigated the influence of two factors (familiarity and cues vs. no cues) on listener attitudes towards speakers with dysarthric speech, two hypotheses were formulated. Firstly, familiar listeners will have significantly higher ratings pertaining to their attitudes towards communicating with dysarthric speakers on the cognitive, affective and behavioural components of attitude. Secondly, it is hypothesized that the use of speech supplementation strategies will yield higher ratings on the cognitive, affective and behavioural components of attitude, than the use of no cues.

\section{Research Design}

A comparative research design was employed for the study (Schiavetti \& Metz, 2006). This design was selected as it was appropriate to investigate the differences between the attitudes of the two groups of listener participants (familiar vs. unfamiliar) towards speakers with mixed dysarthria who use speech supplementation strategies.

\section{Participants}

The study involved the use of two participant groups. 
The speakers were individuals with MND that presented with mixed dysarthria who produced speech samples. The second participant group included listeners that were either familiar or unfamiliar with communicating with a person with communication disorders.

\section{Speakers with dysarthria}

Three individuals with MND that presented with mixed dysarthria participated in the study. Using a non-probability, purposive sampling strategy (Schiavetti \& Metz, 2006), speakers that met the following selection criteria were recruited with the assistance from the Motor Neuron Disease Association (Gauteng): (i) diagnosis of MND made by a certified neurologist; (ii) used speech as their primary mode of communication; (iii) English first language speaker; (iv) reading ability at or above the $5^{\text {th }}$ grade level; (v) presented with mixed dysarthria and in the $3^{\text {rd }}$ or $4^{\text {th }}$ stage of dysarthria (Mathy et al., 2000) (Appendix A); (vi) speech intelligibility scores between $30-70 \%$ as measured by the Sentence Intelligibility Test (SIT) (Yorkston, Beukelman \& Tice, 1996), and; (vi) able to produce at least eight consecutive words in connected speech. See Table 1 for detailed information regarding each speaker.

\section{Table 1. Demographic information for the speakers} with dysartbria

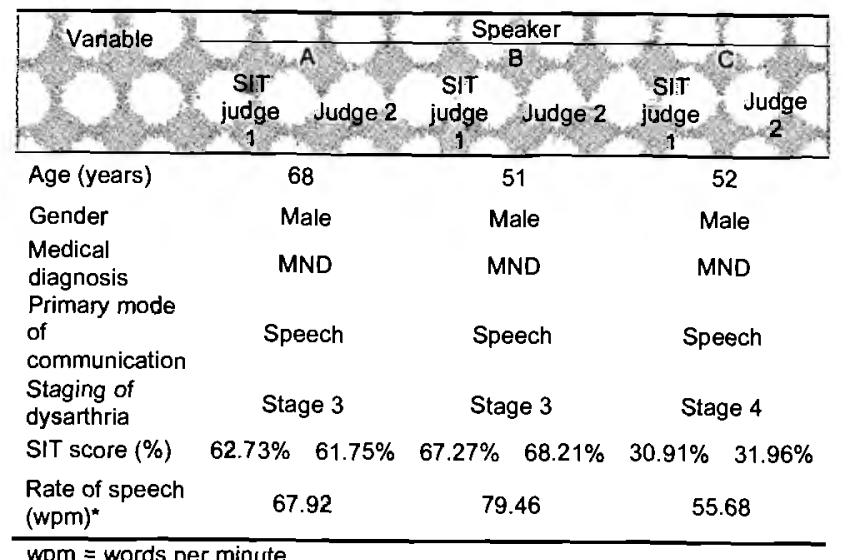

wpm $=$ words per minute

\section{Listener Participant Group}

The primary participants in this study were the listeners. A total of 36 participants were recruited for the study. A non-probability sampling strategy, 'purposive sampling' was employed to recruit the 18 familiar listeners (FL), and 'convenience sampling' to recruit the 18 unfamiliar listeners (UL). The listener participant groups met the following criteria: (i) aged 30 - 75 years; (ii) English first language speaker; (iii) no self-reported hearing loss; (iv) no identified language, learning, cognitive or visual disabilities per self report. Familiar listeners were defined as individuals (family member/friend/care worker) who had experience in communicating, on a daily basis with a person with a communication disorder (Hustad, 2001a,b). Unfamiliar listeners were defined as individuals that had no more than incidental experience listening to and communicating with persons having communication disorders. The two listener groups were matched for gender and mean age. The unfa- miliar listener group comprised of 18 participants (14 female, 4 male). Their average age was 58.0 years (range 32 to $75 ; \mathrm{SD}=10.3$ ). The familiar listener group recruited through the Parkinson's disease Association support group of South Africa, comprised of 18 listeners (14 female, 4 male). Their average age was 50.7 years (range 32 to $79 ; \mathrm{SD}=14.8$ ). Demographic information regarding listeners assigned to each listener group is provided in Table 2.

\section{Table 2. Demographic information for the listener groups}

\begin{tabular}{ccccc}
\multirow{2}{*}{$\begin{array}{c}\text { Group } \\
\text { participant }\end{array}$} & \multicolumn{2}{c}{$\begin{array}{c}\text { Eamiliar } \\
\text { Listener }\end{array}$} & \multicolumn{2}{c}{$\begin{array}{c}\text { Unfamiliar } \\
\text { Listener }\end{array}$} \\
\cline { 2 - 6 } & Age & Gender & Age & gender \\
\hline 1 & 56 & Female & 53 & Female \\
2 & 56 & Female & 73 & Female \\
3 & 58 & Female & 55 & Female \\
4 & 66 & Female & 42 & Female \\
5 & 65 & Female & 40 & Male \\
6 & 71 & Female & 69 & Female \\
7 & 62 & Female & 71 & Female \\
8 & 38 & Female & 62 & Female \\
9 & 57 & Female & 38 & Female \\
10 & 54 & Female & 79 & Female \\
11 & 56 & Female & 37 & Female \\
12 & 32 & Female & 61 & Female \\
13 & 58 & Male & 42 & Female \\
14 & 68 & Female & 32 & Male \\
15 & 55 & Male & 38 & Female \\
16 & 57 & Male & 36 & Male \\
17 & 60 & Female & 44 & Female \\
18 & 75 & Male & 42 & Male \\
\hline
\end{tabular}

\section{Materials}

\section{Speech Stimuli}

Four narrative passages, each consisting of 10 sentences were employed as speech stimuli. Narratives (Appendix B) representing situational information common to first language English speakers were developed based on linguistic characteristics specified in a study conducted by Hustad (2001b). The length and content of the stimulus material were equalised across the following linguistic parameters: (i) sentences per narrative; (ii) topic per narrative; (iii) words per narrative; (iv) number of words per sentence; and (v) reading level. Summary statistics for stimulus material are presented in Table 3.

\begin{tabular}{cc} 
Table 3. Linguistic characteristics of narratives \\
\hline \multicolumn{1}{c}{ Characteristic } & Number \\
\hline Sentences per narrative & 10 \\
Topics per narrative & 1 \\
Words per narrative & 65 \\
5-word sentences per narrative & 2 \\
6 word sentences per narrative & 3 \\
7 word sentences per narrative & 3 \\
8 word sentences per narrative & 2 \\
Reading level for each narrative & 5 \\
\hline
\end{tabular}

\section{Questionnaire}

A questionnaire (Appendix C) to measure the primary dependant variables was developed, and included demographic information and three statements probing listener 
attitudes in the cognitive, behavioural and affective domains. These statements were selected as they directly address the three components of attitude and were the same as those employed in the other studies (Hustad, 2001b; Hustad \& Gearhart, 2004). Each of the three variables were measured using a 7 -point ordinal Likert scale, with $1=$ strongly disagree and $7=$ strongly agree. Listeners were required to circle the number which most closely represented their perception of each speaker. The statements were: (i) I think this person is an effective communicator using this strategy; (ii) I would feel comfortable communicating with this person in a class or at work if $\mathrm{s} / \mathrm{he}$ used this strategy; and (iii) I would be willing to communicate with this person in a class or at work if $s /$ he used this strategy. In addition a qualitative question ("What feelings would you experience if you were to communicate with the speakers?") was included to obtain information regarding the listener's feelings/emotions towards the speaker after hearing the speaker.

\section{Data collection procedures}

\section{Ethical considerations}

Various ethical considerations were implemented throughout the research study. The researcher obtained ethical clearance from the Human Research Ethics Committee (medical) of the University of the Witwatersrand before this research study was implemented. Written informed consent was obtained from the MND Association, Parkinson's disease Association and all participants, using established and approved methods. All participants in the study were fully informed of the nature of the study and were assured of anonymity and confidentiality. Each participant was required to sign a consent form, providing proof of his/her willingness to partake in the study and had the right to withdraw from the study at any time, without suffering any negative consequences.

Data collection from the speakers with dysarthria: Recording speech samples

The recording of narrative samples took place in a quiet room in the speakers' homes, and were obtained using a Sony Mini-Disc Walkman (MZ-R900) digital audio recorder and a head mounted Sennheiser microphone to limit disturbance noises during the recording as a result of microphone movement. The head mounted microphone helped to maintain a constant distance from the speaker's mouth so as to obtain correct'sound levels during the recording.

Each speaker was instructed to read all four narrative passages, printed in font size 20 and presented directly in front of the speaker, as naturally as possible to emulate a typical communication situation. The rate and prosody of the speakers was not controlled.

Constructing the stimuli (audio-visual footage)

The recorded speech samples were transferred digitally to a Toshiba Satellite (A100-519) laptop via the onboard sound card. The recordings were edited using digital audio editing software (Acoustica v4.0). The speech samples were edited to remove extraneous comments, and the amplitude of all the samples normalized to a $-5 \mathrm{~dB}$ setting using the sound editing software to ensure that all the speech samples were at a similar volume level.

The edited speech samples were then combined with visual information to implement the combined topic-alphabet cue strategy using Windows Movie Maker software (version 6 , Windows Vista OS). The visual orthographic information took the form of a text presentation on the screen showing the narrative topic cue, and the display of the first alphabet letter of each word spoken during the narrative. The display of the alphabet cue visuals was synchronized to the actual speech sample, so that as the speaker produced each word, the first letter was displayed simultaneously in real-time. For the stimuli where no cues were used, a blank screen was displayed in the background whilst the audio speech sample was played.

\section{Presentation of stimuli to the listeners}

The final step involved the presentation of the compiled stimuli to the unfamiliar (UL) and familiar (FL) groups. The audio-visual recordings containing the narrative samples of the three speakers associated with the combined cue condition and the no cues condition were presented to the 36 listeners.

All 36 listeners viewed the audio-visual footage in a quiet room at their place of residence. All the listeners were seated during the viewing with the laptop positioned directly $(0.5 \mathrm{~m})$ in front of them. The volume was normalized at the maximum output of the laptop for each listener, so that all the listeners listened to the stimuli at the same sound level.

Listeners were instructed that they would hear two speakers each reading a narrative, one purely auditory without any supplementation cues and the other with the combined alphabet and topic cue. The listener was briefed prior to the presentation on how the combined supplementation strategy worked and how it would appear on the screen.

Following the completion of the listening task, all participants were requested to independently complete the questionnaires after receiving verbal and written instructions on the requirements of the questionnaire.

\section{Randomization}

To prevent the possibility of order and learning effect of cue conditions during the presenting of the narrative audiovisual stimuli to the listeners, the sequence of the cue/no cue narratives shown to each listener was alternated. Different sequences and combinations of possible presentation orders for cues condition, speakers ( 3 speakers) and narratives (4 narratives) was used in the study. Each listener in the unfamiliar listener group was matched to one of the 18 in the familiar listener group. Therefore 18 sequences were displayed in total, ensuring that no two listeners were exposed to the same speaker, narrative, and cue combination.

\section{Data Analysis}

The values obtained from the Likert-type scales were recorded and collated for statistical analysis. Descriptive statistics were utilized to summarize and organize the data collected. A two-way repeated measure Analysis of Variance (ANOVA) design was used (one-factor repetition) to determine the effect of the two experimental ('familiarity' and 'cue/no cue') factors on the listeners' attitudes.

The design used a within-subjects repeated measure for the 'cues/no cues' factor in the design, since the listener attitude rating measurements for both the cues and no-cues condition were obtained from the same listener participants. Two different sample groups for the familiar and non-familiar listeners were used, and therefore repeated-measures 


\section{NATALIE TOY AND KARIN JOUBERT}

were not used for the 'familiarity' factor of the listeners in the research design.

The research design was employed for each of the three listener attitude components measured in the study. The three dependant variables were the listener ratings of communication effectiveness (cognitive); listener feelings and emotions (affective); and willingness/persistence to communicate and understand the speaker (behavioural).

\section{Results and Discussion}

The results of the study will be described and discussed in this section in relation to the aims of the research. The results from the research data indicated mean listener attitude ratings across all listener and cue conditions (combined cues and no-cue strategy) of $M=3.241(S D=1.852$ ) for the cognitive component; $M=3.612(S D=1.936)$ for the affective component; and $M=4.00(S D=1.933)$ for the behavioural component.

\section{Listener Attitude Ratings}

\section{Communicative effectiveness}

The mean attitude ratings for the communicative effectiveness of listener attitude are shown in Figure 1 below. The figure shows a difference in the cognitive attitude rat-

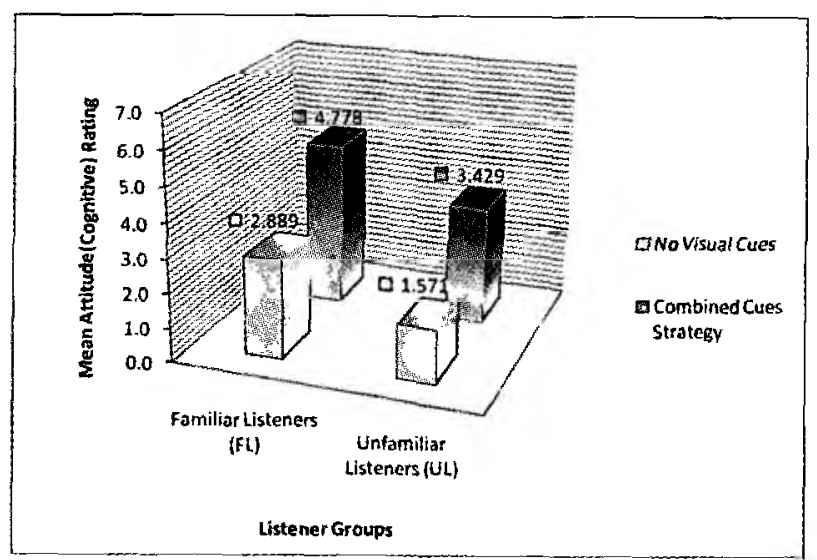

Figure 1. Mean Attitude (cognitive component) ratings for the listener groups (Familiar, Unfamiliar) with Combined

ings (mean) for the familiar listener group (Combined cues: $M=4.77, S D=1.47$; No Cues: $M=2.88, S D=1.52$ ) and the unfamiliar listener group (Combined Cues: $M=3.42, S D=1.55$; No Cues: $M=1.57, S D=1.50)$. The difference exists for both

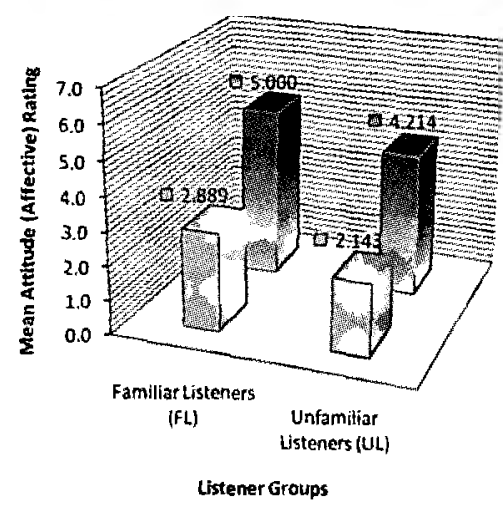

GNa Visual Cues

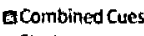
Strategy

Figure 2. Mean Attitude (affective component) ratings for the listener groups (Familiar, Unfamiliar) with Combined Cues, No Cues condition the combined cues and no cues condition.

Listener emotions and feelings (affective)

The mean attitude ratings for the affective attitude component (see Figure 2) show similar results to those observed for communication effectiveness, with a slight difference observed in the ratings between the familiar listener ( $\mathrm{Com}$ bined Cues: $M=5.00 S D=1.47$; No Cues: $M=2.88 S D=1.52$ ) and unfamiliar listener group (Combined Cues: $M=4.21$ $S D=1.55$; No Cues: $M=2.143 S D=1.50$ ). Again this difference is observed for both the cue conditions.

Listener willingness and persistence to communicate (behavioural)

The results for the behavioural component (Figure 3) of the listener attitudes showed slightly higher mean ratings than the other attitude components. As observed in the pre-

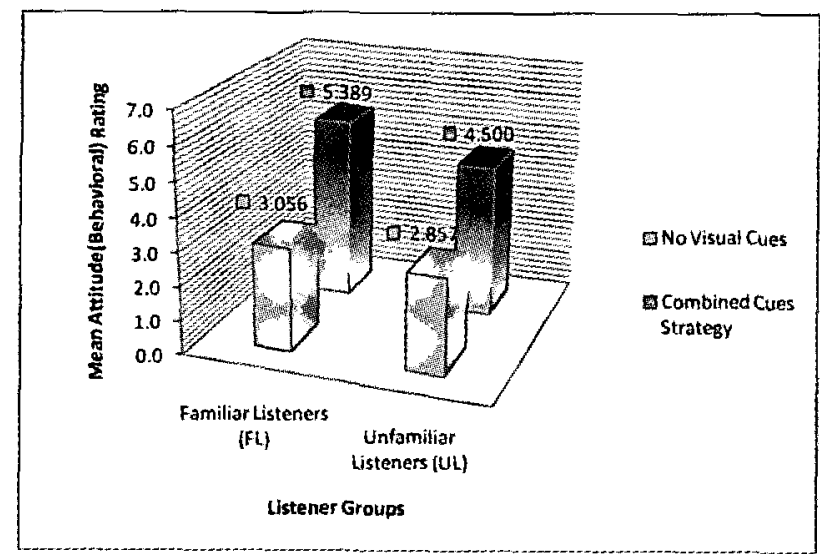

Figure 3. Mean Attitude (behavioral component) ratings for the listener groups (Familiar, Unfamiliar) with Combined Cues, No Cues condition

vious attitude component results, the behavioural attitude results show a slight difference between the familiar listener (Combined Cues: $M=5.38$ SD $=1.47$; No Cues: $M=3.056$ $S D=1.52$ ) and unfamiliar listener groups (Combined Cues: $M=4.5 S D=1.55$; No Cues: $M=2.85 S D=1.50$ ).

A two-way repeated measure Analysis of Variance (ANOVA) test with one factor repetition for the 'cues/no cues' treatment was performed for each of the three dependant measures. The test assumes that the treatment factors have equal variances (this assumption was confirmed for each set of dependent measure data by performing an equal variance test). The results of the test are described for each treatment factor ('Familiarity' and 'Cues condition') in the following section.

\section{Listener Familiarity}

Hypothesis 1 stated that familiar listeners will have significantly higher ratings than unfamiliar listeners, pertaining to their attitudes towards the speech of dysarthric speakers, measured for the cognitive, affective and behavioural components of attitude. The results of this hypothesis, when tested using a two-way repeated measure ANOVA to compare the mean difference on the attitude ratings for FL as compared to UL is presented in Table 4.

Results of testing hypothesis 1

The two-way repeated measure ANOVA tests applied to all 3 dependent measures (Table 4) indicates that the differences observed in mean values for the 'listener familiarity' 
Table 4. Two way repeated measure ANOVA (One Factor Repetition) results for Listener Attitude (Cognitive, Affective E Behavioral components)

Dependent Variable Listener Attitude(Cognitive)-Communicative Effectiveness Equal Variance Test: Passed $(P=0.596)$

$\begin{array}{lcccc}\text { Source of Varialion } & \text { SS } & F & P & \begin{array}{c}\text { Power/Test } \\ \text { Sensitivity }\end{array} \\ \text { Familiarity } & 0.374 & 0.121 & \mathbf{0 . 7 3 0} & 0.050 \\ \text { Cue/No Cue } & 68.881 & 29.796 & <\mathbf{0 . 0 0 1} & 1.000 \\ \text { Familiarity } \times \text { Cue/No Cue } & 0.00620 & 0.00268 & \mathbf{0 . 9 5 9} & 0.050\end{array}$

Comparisons for factors * (Overall significance level $=0.05$ )

\begin{tabular}{|c|c|c|c|c|}
\hline & $\begin{array}{l}\text { Difference of } \\
\text { Means }\end{array}$ & Unadjusted P & Critical Level & Significant \\
\hline FL vs. UL & 0.444 & 0.730 & 0.050 & NO \\
\hline Combined Cues vs. No Cues & 2.091 & 0.0169 & 0.050 & YES \\
\hline Equal Variance Test: & Passed $(P=0$ & & & \\
\hline Source. of Variation & sS & $F$ & $\boldsymbol{P}$ & $\begin{array}{l}\text { Power/ Test } \\
\text { Sensitivity }\end{array}$ \\
\hline Familiarity & 0.211 & 0.0761 & 0.785 & 0.050 \\
\hline Cue/No Cue & 55.254 & 30.278 & $<0.001$ & 1.000 \\
\hline Familiarity $\times$ Cue/No Cue & 0.00397 & 0.00217 & 0.963 & 0.050 \\
\hline
\end{tabular}

Comparisons forfactors * (Overall significance level $=0.05$ )

\begin{tabular}{lcccc}
\hline & $\begin{array}{c}\text { Difference of } \\
\text { Means }\end{array}$ & Unadjusted P & Critical Level & Significant \\
FL vs. UL & $\mathbf{0 . 3 3 3}$ & 0.785 & 0.050 & NO \\
Combined Cues vs. No Cues & $\mathbf{1 . 8 7 3}$ & 0.0161 & 0.050 & YES
\end{tabular}

Tistener Attitude(Behavioral) - Eistener's willingness to communicate
Equal Variance Test: $P$ assed $(P=1.000)$

\begin{tabular}{lcccc}
\multicolumn{1}{c}{ Source afVariation } & SS & $F$ & $\boldsymbol{P}$ & $\begin{array}{c}\text { Power/ Test } \\
\text { Sensitivity }\end{array}$ \\
Familiarity & 0.988 & 0.294 & $\mathbf{0 . 5 9 2}$ & 0.050 \\
Cue/No Cue & 100.829 & 27.624 & $<\mathbf{0 . 0 0 1}$ & 1.000 \\
Familiarity x Cue/No Cue & 62.252 & 0.833 & $\mathbf{0 . 3 6 9}$ & 0.050
\end{tabular}

Combarisons for factors * (Overall significance level $=0.05$ )

\begin{tabular}{|c|c|c|c|c|}
\hline$f$ & $\begin{array}{l}\text { Difference of } \\
\text { Means }\end{array}$ & Unadjusted P & Critical Level & Significant \\
\hline FL vs. UL & 0.722 & 0.592 & 0.050 & NO \\
\hline Combined Cues vs. Wo Cues & 1.988 & 0.0210 & 0.050 & YES \\
\hline
\end{tabular}

Type I, $\alpha=0.050$

* Pair wise Multiple Comparison Procedures (Holm-Sidak method)

factor is not sufficiently.large to exclude the possibility that the difference is due to random sampling variance, when the effects of the other factor (combined cues versus no cues strategy) is taken into account. Comparison of the FL and UL groups yielded mean differences between the groups of 0.444 for the cognitive component $(P=0.730)$; 0.333 for the affective component $(P=0.785)$; and 0.722 for the behavioural component $(P=0.592)$. Therefore there is no statistically significant difference in the scores of the FL sample group as compared to the UL sample group, across all 3 listener attitudes.

The study results therefore conclude that the hypothesis stating that 'familiarity' plays an influencing factor on the attitude ratings (all three components) of listeners is not valid. The effect of familiarization of the listener in influencing their attitudes towards dysarthric speakers would therefore appear to play no significant role. This result is however in contrast to the results indicated by the longitudinal study carried out by De Paul and Kent (2000), which concluded that a familiar listener had superior performance over unfamiliar listeners. According to their study, the performance was significantly improved especially during the individuals' second year of progression of MND when their speech intelligibility decreased markedly.

Several factors may have contributed to the current results. The familiar listener participant group was defined as listeners who had experience in communicating on a daily basis with persons with a communication disorder. However, it did not specify the degree of the dysarthria of the person that these familiar listener individuals communicated with or had contact with. In this study, the familiar listener participants were 'familiar' with individuals who had Parkinson's disease. It was noted that many of the familiar listeners communicated with individuals in the early stages of Parkinson's disease, where their speech was only mildly impaired. These 'familiar' listener participants would therefore not have any substantial experience in communicating with speakers who had severe dysarthria.

In this study the duration of interaction between the familiar listener and speaker was not specified and this variability of exposure was not taken into account. The familiar listener may have interacted with the individual for a month or a few years. This is in contrast to the longitudinal case study done by De Paul and Kent (2000). Therefore, the researchers' selection criteria had to be stricter in specifying the frequency and length of exposure to an individual with dysarthria, as well as the severity of dysarthria. However the researchers were unable to define the selection criteria to include exposure and stricter familiarity requirements for the study due to the nature and time limitations of the research.

Speech Supplementation Strategy (Combined cues vs. No cues)

Hypothesis 2: The use of a speech supplementation strategy, in this case the combined topic and alphabet cues will yield higher ratings, than the use of no cues pertaining to listeners' attitudes towards the speech of dysarthric 
speakers.

\section{Results of testing hypothesis 2}

The results of the two-way repeated measure ANOVA (one factor repetition) analysis are presented in Table 4 in the previous section. This analysis compared the mean difference on the listener attitude ratings for the use of the combined topic and alphabet cue, as compared to the use of no cues pertaining to their attitudes towards the speech of dysarthric speakers. Three components of attitude were measured namely, cognitive, affective and behavioural.

Communicative effectiveness (cognitive): The two-way repeated measure ANOVA determined that there was a statistically significant difference $(P=<0.001)$ in the mean values among the different levels of cues. The difference in the mean values among the different levels of cues is greater than would be expected by chance after allowing for the effects of differences in familiarity.

The two-way repeated measure revealed ratings of effectiveness that were significantly higher for combined cues than for the no cue condition. All the statistical findings for ratings of communication effectiveness in this study are consistent with intelligibility data obtained in previous studies (Hustad, 2003; Hustad \& Beukelman, 2003; Hustad et al., 2003). The indication is that the combined topic and alphabet cue results in more favourable ratings of effectiveness and intelligibility than the no cue condition.

According to Hustad (2001b), a reason for the positive effect of the combined cue on effectiveness rating may be that all the listeners recognized attempts to enhance speech via the strategy presentation and they equated the use of the multiple supplementation strategies as trying "harder" to compensate to a greater extent for the communication difficulties.

Listener comfort and emotion (affective): Similarly, the two-way repeated measure ANOVA determined that there was a statistically significant difference $(P=<0.001)$ in the mean values among the different levels of cues. As was the case for listener effectiveness combined cues yielded more favourable affective attitude ratings than the no cue conditions. The results suggest that the more information the listener has about a topic the more comfortable they would be to interact with the speakers. Participants stated that because they were provided with the topic of the narrative, as well as the alphabet cues, they were able to anticipate and understand more of what the speaker was saying.

Listener persistence (behavioural): Again, the two-way repeated measure ANOVA determined that there was a statistically significant difference $(P=<0.001)$ in the mean behavioural rating. Listeners are therefore more persistent to interact with speakers that employed the combined topic and alphabet cue supplementation strategy in conjunction with their habitual speech, as compared to the no cue con- dition.

The overall results of the present study showed that the UL and FL sample groups were from the same population group and therefore listener familiarity was not a favourable indicator of increased intelligibility and listener attitude ratings. However, the present study did show that the combined alphabet and combined cue supplementation strategy used in conjunction with habitual speech, resulted in sig- nificantly higher ratings of listeners' perceptions of the communication of speakers with dysarthria ranging from $33 \%$ - $67 \%$ intelligibility.

It is however important to consider these findings in light of the limitations of the research. The study employed a traditional experimental paradigm which differs significantly from naturalistic, real life communication situations. As the speakers and listeners did not converse and interact with one another in communicative exchanges, extra-linguistic factors such as gesture, body language and facial expression used in conjunction with verbal communication were omitted. The listeners were also not able to directly interject and ask for clarification from the speaker.

The content, form and use of messages produced by the speaker were also scripted, and the environment in which the listeners heard the audio recordings was manipulated for an ideal listening and viewing condition. The audio-visual material presented to the listeners for viewing and listening was digitally enhanced, so that all the cues used were clearly and readily visible. In a real life situation and implementation of these supplementation strategies, the communicative partner does not have the advantage of this type of digital enhancement. The communicative partner would have to deal with the effects of lighting, distance from the speaker and the angle at which the partner is facing towards the speaker. In addition the speech patterns of the speaker may have been different if they were directly implementing the supplementation strategy, for example slowing down of their speech rate as they point to the various alphabet cues, thus affecting their overall speech intelligibility as perceived by the listener. These factors need to be considered in light of the current research.

The sample size of the study involved only 3 speakers with dysarthria and 36 listener participants; therefore this relatively small sample size may have an influence on the reliability and validity of the study. In addition the participants in the study were restricted to a specified age range. The results indicated by the study may not be fully representative of the general population.

In this study, only three Likert-type questions were used to gather information regarding listener attitudes. Although the construct of attitude and its constituent components (cognitive, affective and behavioural) are well grounded in literature (Hustad \& Gearhart, 2004), measurement of these constituents in a research study is more complex. The measurement of listener attitudes based on three questions cannot fully account for the many different facets of a listener's attitude and it is suggested that future research should aim to adapt or develop a more comprehensive instrument with well-described psychometric properties to explore listener attitudes.

The results of the qualitative question: "What feelings would you experience if you were to communicate with the speakers?" indicated a few general trends across all 36 listeners' emotional reactions towards the 3 speakers. The majority of listeners felt a great sense of empathy and awkwardness towards the speakers because they acknowledge the speakers' intent to communicate, but still struggled to understand them. Listeners stated a great sense of awkwardness in not knowing what the speaker is saying and not being able to re- 
spond appropriately during a communicative exchange.

Listeners also stated becoming highly frustrated with the speakers and frequently becoming quite impatient. Most of the participants stated that it would have been easier to understand what the speaker was saying, if they were face-toface with the speaker in comparison to only being able to listen to a recording of their speech.

The majority of the participants did indicate that they found the cues beneficial in helping them to understand the speaker. However, a few listener participants did state that they found the visual cues misleading, stating they had difficulty in focusing on the cues and integrating the visual and auditory stimuli (this was particularly evident when the speaker spoke at a fast rate).

\section{Conclusion}

The overall aim of the study was to determine the influence of familiarity on a listener's attitude towards speakers with mixed dysarthria. The findings of this study concluded that familiarity has no statistically significant influence on their attitudes towards speakers with mixed dysarthria.

The results, however did suggest that speakers whose speech intelligibility is severely reduced may benefit markedly from using a combined topic-alphabet cue strategy, as within this experimental research paradigm, the combined cue strategy resulted in more favourable listener attitudes. In addition, listeners appeared to be more comfortable and persistent in communicating with speakers who implemented the combined cues. Although many variables are likely to influence listener attitudes, there is strong evidence to suggest that listener attitudes are closely linked to intelligibility (Hustad \& Gearhart, 2004).

The findings of the influence of familiarity on the listener attitudes may possibly have been affected by the time constraints imposed on the study and an insufficient definition of the familiar listener. It is suggested that the definition of a familiar listener include specific detail on the duration and frequency of interaction with the person with dysarthria as well as the severity of the reduction in the speaker's intelligibility. Other studies (DePaul \& Kent, 2000) indicated that familiarity does lead to significant performances on speech intelligibility and therefore listener attitudes. It further indicated that an advantage of familiarity evolved over time and reached a maximum level when the speech impairment was significantly reduced in intelligibility (DePaul \& Kent, 2000).

The results of the study should be interpreted in light of the experimental nature of the study where the listeners did not communicate in a real communicative exchange and it therefore does not permit generalisation to ecologically valid communication situations.

This study however has important implications in the clinical management of individuals with motor speech disorders, such as dysarthria. The implementation of speech supplementation strategies such as the use of combined alphabet-topic cues can improve the communicative effectiveness and lead to more favourable interactions with listeners.

Future research should address the attitudes of unfamiliar and familiar listeners towards individuals with different severity levels and speech characteristics, using more natu- ralistic speech samples. It is important that future research should take intra-rater reliability into account by repeating a small proportion of speech samples. In addition, a qualitative approach could be employed to investigate how AAC strategies affect listener attitudes to provide information regarding what listeners think, feel and believe when faced with speakers using AAC.

\section{References}

Antonak, R., \& Livneh, H. (1988). The measurement of attitudes towards people with disabilities. Charles $\mathrm{C}$. Thomas: Springfield, IL.

Beliveau, C., Hodge, M., \& Hagler, P. (1995). Effects of supplemental linguistic cues on the intelligibility of severely dysarthric speakers. Augmentative and Alternative Communications, 11, 176-186.

Beukelman, D. R., Yorkston, M., \& Reichle, J. (2000). Augmentative and alternative communication for adults with acquired neurological disorders. Brooks Publishing Co.: Baltimore.

Beukelman, D., \& Yorkston, K. (1977). A communication system for the severely dysarthric speaker with an intact language system. Journal of Speech and Hearing Disorders, $42,265-270$.

Britnell, E. S., Madill, H. M., Montgomerie, T. C., \& Stewin, L. L. (1992). Work and family after injury: Do female and male client perspectives differ? Career development quarterly, 41, 145-160.

Crow, E., \& Enderby, P. (1989). The effects of an alphabet chart on the speaking rate and intelligibility of speakers with dysarthria. In K. Yorkston, \& D. Beukelman (Eds), Recent advances in clinical dysartbria (pp. 99-108). PROED: Austin, TX.

DePaul, R., \& Kent, R. D. (2000). A longitudinal case study of ALS: effects of listener familiarity and proficiency on intelligibility judgements. American Jounal of Speech-Language Patbology, 9 (3), 230-240.

Dongilli, P. (1994). Semantic context and speech intelligibility. In J. Till, K. Yorkston, \& D. Beukelman (Eds), Motor Speech Disorders: Advances in assessment and treatment (pp. 175-191). Paul H. Brookes Publishing Co.: Baltimore.

Duffy, J. R. (2005). Motor speech disorders: substrates, differential diagnosis and management. Mosby: St. Louis.

Eiser, J. R. (1986). Social Psychology: Attitudes Cognition and Social Behaviour. Cambridge University Press: Cambridge, UK.

Fox, L., Sohlberg, M. M., \& Fried-Oken, M. (1999). Effects of conversational topic self selection on augmentative communication intervention for adults with aphasia. Paper presented at the Clinical Aphasiology Conference. Keywest, FL.

Freed, D. (2000). Motor Speech Disorders: Diagnosis and treatment. Singular Publishing Co.: San Diego.

Garcia, J., \& Cannito, M. (1996). Influence of verbal and non-verbal contexts on the sentence intelligibility of a speaker with dysarthria. Journal of Speech and Hearing Research, 39, 750-760.

Greenwald, A. G., Brock, T. C., \& Ostrom, T. M. (1968). Psychological foundations of attitudes. Academic Press: 
NY.

Hunter, L., Pring, T., \& Martin, S. (1991). The use of strategies to increase speech intelligibility in cerebral palsy: An experimental evaluation. British Journal of Disorders of Communication, 26, 163-174.

Hustad, K. C. (2001a). Effects of linguistic cues and stimulus cohesion on intelligibility of severely dysarthric speech. Journal of Speech, Language and Hearing Research, 44 (3), 497-510.

Hustad, K. C. (2001b). Unfamiliar listeners' evaluation of speech supplementation strategies for improving the effectiveness of severely dysarthric speech. Augmentative and Alternative Communication, 17 (4), 213-220.

Hustad, K. C. (2003). Effects of presentation mode and repeated familiarization on intelligibility of dysarthric speech. American Journal of Speech-Language Pathology, 12 (2), 198-208.

Hustad, K. C., \& Beukelman, D. R. (2001). Effects of linguistic cues and stimulus cohesion on intelligibility of severely dysarthric speech. Journal of Speech, Language and Hearing Research , 44 (3), 407-510.

Hustad, K. C., \& Beukelman, D. R. (2002). Listener comprehension of severely dysarthric speech: Effects of linguistic cues and stimulus cohesion. Journal of Speech, Language and Hearing Research, 45 (3), 545-558.

Hustad, K. C., \& Cahill, M. A. (2003). Unfamiliar listeners' evaluation of speech supplementation strategies for improving the effectiveness of severely dysarthric speech. Augmentative and Alternative Communication , 4, 213220.

Hustad, K. C., \& Garcia, J. M. (2005). Aided and unaided speech supplementation strategies: Effect of alphabet cues and iconic hand gesture on dysarthric speech. Journal of Speech, Language and Hearing Research, 48 (5), 9961012.

Hustad, K. C., \& Gearhart, K. J. (2004). Listener attitudes towards individuals with cerebral palsy who use speech supplementation strategies. American Journal of SpeechLanguage Pathology, 13 (2), 168-181.
Hustad, K. C., Jones, T., \& Dailey, S. (2003). Implementing speech supplementation strategies: Effects on intelligibility and speech rate of individuals with chronic severe dysarthria. Journal of Speech, Language and Hearing Research , $46(2), 462-474$.

Lasker, J. P., \& Bedrosian, J. L. (2000). Acceptance of AAC by adults with acquired disorders. In D. Beukelman, K. Yorkston, \& J. Reichle (Eds), Augmentative Communication for adults with acquired neurologic disorders (pp. 107136). Paul H. Brookes: Baltimore.

Light, J. A., McNaughton, D., Gulens, M., Kresman, C., Williams, M., \& Cohen, K. (1999). The mentor programme for individuals who use AAC. Unpublished manuscript. Pennsylvania State University.

Mathy, P., Yorkston, K. M., \& Gutmann, M. L. (2000). Augmentative and alternative communication for adults with acquired neurologic disorders. Brookes Publishing Co.: Baltimore, Maryland.

McMillan, J. H., \& Schumacher, S. (2001). Research in education: A conceptual introduction ( 5 th ed.).Addison Wesley Longman Inc.: New York.

Schiavetti, N., \& Metz, D. E. (2006). Evaluatng research in communicative disorders (5th ed.). Allyn and Bacon: Boston.

Triandis, H. C., Adamopoulos, J., \& Brinberg, D. (1984). Perspective and issues in the study of attitudes. In R. L. Jones (Ed), Attitudes and Attitude change: Theory and practice. Council for Exceptional Children: Reston, VA.

Yorkston, K. M., Beukelman, D. R., Strand, E. A., \& Bell, K. R. (1999). Management of motor speech disorders in children and adults. PRO-ED.: Austin, TX.

Yorkston, K., Beukelman, D., \& Tice, R. (1996). Sentence Intelligibility Test for Macintosh. Lincoln, NE.

Young, J. M., \& McNicoll, P. (1998). Against all odds: Positive life experiences of people with advanced amyotroph ic lateral sclerosis. Health and Social Work, 23 (1), 35-43.

Yunusova, Y., Weismer, G., Kent, R. D., \& Rusche, N. M. (2005). Breath-group intelligibiity in dysarthria: Characteristics and underlying correlates. Journal of Speech, Language and Hearing Research , 48 (6), 1294-1310. 
(Mathy, Yorkston, \& Gutmann, 2000)

\section{Stage 1: No detectable speech disorder}

- Speech of individuals with a spinal presentation of ALS sounds normal.

- Speaker notices a change in function.

- Listeners note no changes in speaking rate, precision or loudness.

Stage 2: Obvious speech disorder with intelligible speech

- Changes in speech are apparent.

- Changes may be more apparent with stress or fatigue.

- Most speakers compensate unconsciously for articulator or respiratory impairment by decreasing their speaking rate and the length of their breath groups.

- Speech at this stage remains easy to understand, although voice quality maybe harsh or breathy or mild articulator problems may be present.

Stage 3: Reduction in speech intelligibility

- Speaking rate, articulation and resonance are impaired and may make speech difficult to understand, depending on the communication environment.

- Helpful strategies include maintaining a slower speaking rate, conserving energy, increasing the precision of speech production and developing strategies to resolve communication breakdowns.

- Individuals can do with modifying their speech production (Kennedy, Strand, \& Yorkston, 1994).

- Some speakers begin to use AAC techniques to resolve breakdowns. When speaking rate is $50 \%$ or less, AAC assessment and intervention should be initiated.

- Helpful strategies include maintaining a slow speaking rate, conserving energy, increasing precision of speech production, and developing strategies to resolve communication breakdown.

Stage 4: Natural speech supplemented with augmentative communication

- $\quad$ Speech must be combined with AAC approaches (Kazandijan, 1997).

- Natural speech may be limited to highly predictable messages, such as responses to questions and greetings.

- The speaker must supplement natural speech by writing key words or pointing to the first letter of each word he or she speaks.

- Intervention for speakers at this stage may include alphabet supplementation, changing communication modes for different situations, an alerting signal for gaining attention, augmented telephone communication, and portable writing systems (Hustad, 1999)

Stage 5: No useful speech

- Speakers with advanced bulbar ALS lose speech function.

- Some individuals at this stage may vocalize for emotional expression or with extreme effort but do not produce understandable speech.

- Intervention for speakers at this stage may include establishing reliable yes/no system, eye-gaze systems and communication systems for speaker's dependant on ventilators, and integrated multipurpose AAC systems 


\section{APPENDIX B}

\section{Narrative Stimuli}

\section{Narrative 1: A holiday}

It was an amazing holiday (5). We gathered shells on the beach (6). We also saw dolphins swimming in the sea (8). We spent hours lying in the warm sun (8). One day we hiked up Table Mountain (7). The view was so magnificent and peaceful (7).It was the best experience ever (6). We have a beautiful country (5). The week had come to an end (7). My fun holiday had officially ended (6).

\section{Narrative 2: A sporting event}

The two Batsmen walked onto the cricket field (8). The crowd was cheering wildly (5). Everyone was standing and clapping their hands (7). The sun was shining directly overhead (6). The Umpires briefly spoke to each other (7). They asked the players to begin the match (8). Each player went to their position (6). The crowd started a Mexican wave (6). It went round and round the stadium (7). It was a great game (5).

\section{Narrative 3: My wedding day}

The special day had arrived (5). Finally, my eldest daughter was leaving home (7). It was an exciting day (5). The church hall was decorated with red roses (8). The bridesmaids wore pink satin dresses 6 ). The groom and his best man wore white tuxedo's (8). A silver limo arrived at twelve o' clock (7). The wedding music started to play (6). We walked together down the aisle(6). Then my daughter looked at me and smiled (7).

\section{Narrative 4: The Trip To The Doctor}

I woke up with a fever and headache (8). I could barely sit up (5). I was in such pain and discomfort (7). The whole night I could not sleep (7). My grandmother took me to the doctor's office (8). I waited there for 40 minutes (6). My grandmother was angry and frustrated (6). Finally, I went into the doctor's office (7). I hated going to the doctor (6). I went home and slept (5). 


\section{APPENDIX C}

\section{Attitude Questionnaire}

Date:

Code:

Date of birth:

Sex: Male/ female

Age:

First language:

Highest level of education:

Relationship to the person with Parkinson's disease:

Spouse/ parent/ caregiver/ family friend/other

If OTHER, please describe:

Do you have any hearing problems?

Yes / No

If YES, please describe:

Do you have any language, learning or cognitive problems?

Yes I No

If YES, please describe: 


\section{APPENDIX C (Continued)}

SECTION A

Ithink this person is an effective communicator using this strategy

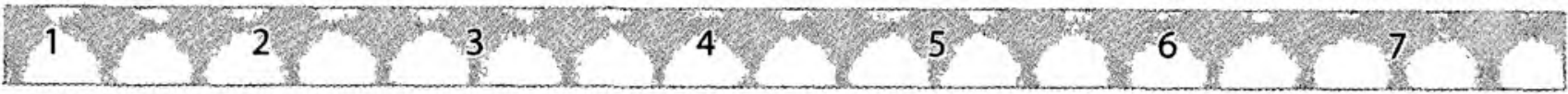

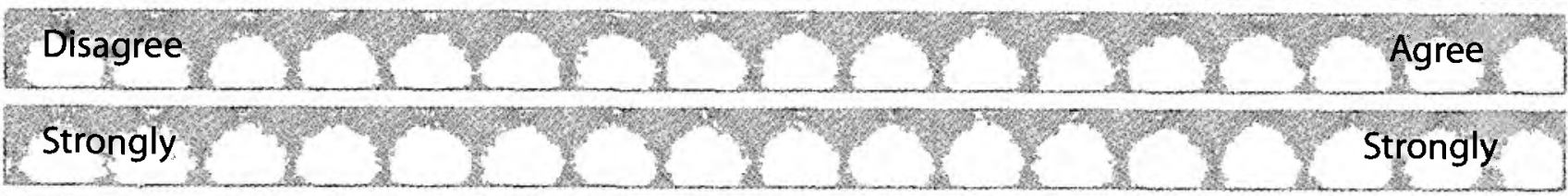

I would feel comfortable communicating with this person in a class or at work if he/she used this strategy

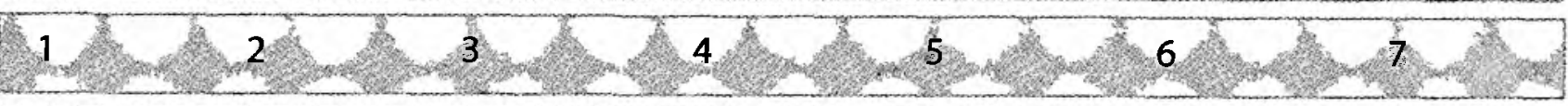

Disagree

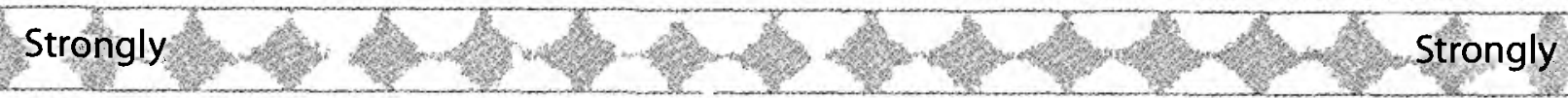

I would be willing to communicate with this person in a class or at work if he/she used this strategy.

1 2 2 ?

$3+4$

5 \% 6

7.2

Disagree

Strongly

Strongly

SECTION B

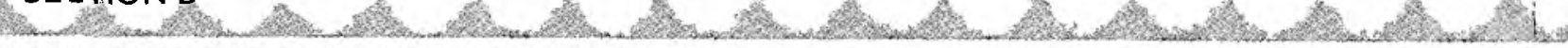

Please complete the following questions truthfully and to the best of your knowledge:

What feelings would you experience if you were communicating to the two previous speak-
ers?

H

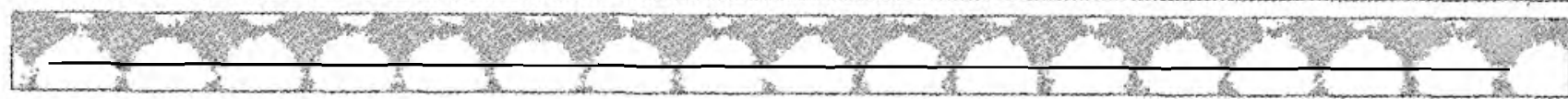

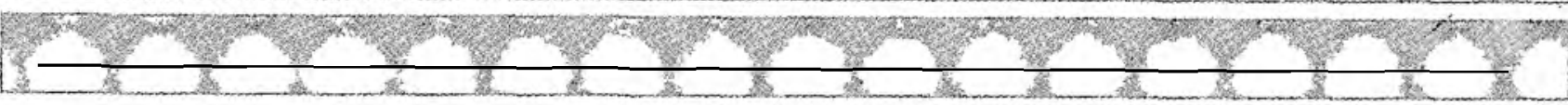

